\begin{tabular}{c} 
International Journal of Advanced Astronomy, $4(1)(2016) 33-38$ \\
SPC \\
International Journal of Advanced Astronomy \\
Website: $\begin{array}{c}\text { www.sciencepubco.com/index.php/IJAA } \\
\text { doi: } 10.14419 / \text { ija. } v 4 \text { Ail.5834 } \\
\text { Research paper }\end{array}$ \\
\hline
\end{tabular}

\title{
Stability of triangular equilibrium points in the Photogravitational elliptic restricted three body problem with Poynting-Robertson drag
}

\author{
Vivek Kumar Mishra*, J.P. Sharma, Bhola Ishwar \\ Department of Mathematics, B.R.A. Bihar University Muzaffarpur-842001 \\ *Corresponding author E-mail:vivek.mishra2626@gmail.com
}

\begin{abstract}
We have examined the stability of triangular equilibrium points in photogravitational elliptic restricted three-body problem with Poynting-Robertson drag. We suppose that smaller primary is an oblate spheroid. We have taken bigger primary as radiating. We have found the location of triangular equilibrium points and characteristic equation of the problem. We conclude that triangular equilibrium points remain unstable, different from classical case.
\end{abstract}

Keywords:Triangular Points; Photogravitational; ERTBP; P-R Drag.

\section{Introduction}

The elliptic restricted three-body problem (ERTBP) is a generalization of the classical restricted three-body problem (RTBP). It describes the three-dimensional motion of a small particle, called the third body (infinitesimal mass) under the gravitational attraction force of two finite bodies, called the primaries. They revolve in elliptic orbit in a plane around their common centre of mass. The infinitesimal mass moves in the plane of motion of the primaries and does not influence the motion of the primaries. ERTBP is more realistic than RTBP, because the orbits of a large number of celestial bodies are elliptic rather than circular. The stability of triangular points in the ERTBP was investigated by Danby [3]. Broucke [1] studied the stability of periodic orbits in the ERTBP. Radiation and oblateness of the primaries also affect the motion of the infinitesimal mass. Many researchers studied the restricted problem taking into account one or both the primaries as oblate spheroids and radiating. Sahoo and Ishwar [11] examined stability of collinear points in the generalized photogravitational ERTBP. Zimovshchikov and Tkhai [14] investigated stability of libration points and resonance phenomenon in the photogravitational ERTBP. A.Narayan and C.R.Kumar [7] studied the effect of photogravitational and oblateness on the triangular Lagrangian points in ERTBP. J.Singh and A. Umar [12] studied the stability of triangular points in ERTBP under radiating and oblate primaries.

Due to radiation, a drag force known as Poynting-Robertson drag, also affect the motion of infinitesimal mass. Poynting [8] stated that the particle, such as, small meteors or cosmic dust were comparably affected by gravitational and light radiation force, as they approach luminous celestial bodies. He also suggested that infinitesimal body in solar orbit suffers a gradual loss of angular momentum and ultimately spiral into the Sun. Robertson [9] modified the theory of Poynting by considering only terms of first order in the ratio of velocity of the particle to that of light. The radiation force is given by.

$$
F=F_{p}\left\{\frac{\vec{R}}{R}-\frac{\vec{V} \vec{R} R}{C \vec{R} R}-\frac{\vec{V}}{C}\right\}
$$

The last two terms constitute Poynting-Robertson (P-R) effect. Wyatt and Whipple [13] have shown that P-R effect has been of very little significance. Chernikov [2] has dealt with the SunPlanet-Particle model and conclude that due to P-R drag triangular points are unstable. Schuerman [10] studied the classical RTBP by including the radiation pressure and P-R effect. Murray [6] investigated location and stability of the five Lagranginan points in the CRTBP when infinitesimal mass is acted by a variety of drag forces. Liou and Zook [5] examined the effect of radiation pressure, P-R drag and Solar wind drag on dust grains trapped in mean motion resonance with Sun-Jupiter in RTBP. Kushvah and Ishwar [4] examined the linear stability of generalized photogravitational RTBP with P-R drag.

The present study aims to examine the motion of the infinitesimal mass in the ERTBP with radiation, oblateness and P-R drag. We suppose that bigger primary is radiating and smaller is an oblate spheroid. It will contribute a lot to understand the effects of eccentricity, radiation, oblateness and P-R drag on the celestial and stellar systems. The motion of a particle in the double stellar system may be of particular interest, because the system forms considerable part of all stellar systems. The results obtained will be useful to future space missions. The results may be applied for placement of large self-contained space colonies into stable equilibrium point at the $\mathrm{L}_{4}$ or $\mathrm{L}_{5}$ Lagrange points.

This paper is divided in five sections. Section (2) contains equations of motion of our problem. In section (3) and (4), we have found location of triangular equilibrium points and examined stability of triangular equilibrium points respectively while section (5) concludes the paper. 


\section{Equations of motion}

We consider two bodies (primaries) of masses $m_{1}$ and $m_{2}$ with $\mathrm{m}_{1}>\mathrm{m}_{2}$ moving in a plane around their common center of mass in elliptic orbit and a third body (infinitesimal mass) of mass $\mathrm{m}$ is moving in a plane of motion of the primaries. Equations of motion of our problem in rotating and pulsating co-ordinate system are given by (Sahoo and Ishwar) [11]

$\mathrm{x}^{\prime \prime}-2 \mathrm{y}^{\prime}=\frac{\partial \Omega}{\partial \mathrm{x}}+\mathrm{F}_{\mathrm{x}}=\mathrm{U}_{\mathrm{x}}$

$\mathrm{y}^{\prime \prime}+2 \mathrm{x}^{\prime}=\frac{\partial \Omega}{\partial \mathrm{y}}+\mathrm{F}_{\mathrm{y}}=\mathrm{U}_{\mathrm{y}}$

$\mathrm{z}^{\prime \prime}=\frac{\partial \Omega}{\partial \mathrm{z}}+\mathrm{F}_{\mathrm{z}}=\mathrm{U}_{\mathrm{z}}$

where the force function

$\mathrm{U}=\frac{1}{\sqrt{1-\mathrm{e}^{2}}}\left[\frac{\mathrm{x}^{2}+\mathrm{y}^{2}}{2}+\frac{1}{\mathrm{n}^{2}}\left\{\frac{(1-\mu) \mathrm{q}_{1}}{\mathrm{r}_{1}}+\frac{\mu}{\mathrm{r}_{2}}+\frac{\mu \mathrm{A}_{2}}{2 \mathrm{r}_{2}^{3}}+\mathrm{W}_{1}\left(\frac{(\mathrm{x}+\mu) \mathrm{x}^{\prime}+\mathrm{yy}^{\prime}+\mathrm{zz}^{\prime}}{2 \mathrm{r}_{1}^{2}}-\right.\right.\right.$ $\left.\left.\left.\mathrm{n} \arctan \left(\frac{\mathrm{y}}{\mathrm{x}+\mu}\right)\right)\right\}\right]$

$\Omega=\frac{1}{\sqrt{1-\mathrm{e}^{2}}}\left[\frac{\mathrm{x}^{2}+\mathrm{y}^{2}}{2}+\frac{1}{\mathrm{n}^{2}}\left\{\frac{(1-\mu) \mathrm{q}_{1}}{\mathrm{r}_{1}}+\frac{\mu}{\mathrm{r}_{2}}+\frac{\mu \mathrm{A}_{2}}{2 \mathrm{r}_{2}^{3}}\right\}\right]$

$\mathrm{F}=\frac{\mathrm{W}_{1}}{\mathrm{n}^{2} \sqrt{1-\mathrm{e}^{2}}}\left\{\frac{(\mathrm{x}+\mu) \mathrm{x}^{\prime}+\mathrm{yy}^{\prime}+\mathrm{zz}^{\prime}}{2 \mathrm{r}_{1}^{2}} \mathrm{n} \arctan \left(\frac{\mathrm{y}}{\mathrm{x}+\mu}\right)\right\}$

$\mathrm{W}_{1}=\frac{(1-\mu)\left(1-\mathrm{q}_{1}\right)}{\mathrm{c}_{\mathrm{d}}}$

Here dash (') represents differenciation with respect to ecentric anomaly $(\mathrm{E})$. The mean motion of our problem is given by

$\mathrm{n}^{2}=\frac{\left(1+\frac{3 \mathrm{~A}_{2}}{2}\right) \sqrt{1+\mathrm{e}^{2}}}{\mathrm{a}\left(1-\mathrm{e}^{2}\right)}$

$\mathrm{r}_{\mathrm{i}}=\left(\mathrm{x}+\mathrm{x}_{\mathrm{i}}\right)^{2}+\mathrm{y}^{2}+\mathrm{z}^{2}(\mathrm{i}=1,2)$

$\mathrm{x}_{1}=-\mu, \mathrm{x}_{2}=1-\mu, \mu=\frac{\mathrm{m}_{2}}{\mathrm{~m}_{1}+\mathrm{m}_{2}}$

Here, $(x, y, z),\left(x_{1}, 0,0\right)$ and $\left(x_{2}, 0,0\right)$ are the coordinate of $m, m_{1}$ and $m_{2}$ respectively. $q_{1}$ is mass reduction factor and $W_{1}$ is P-R drag due to bigger primary $m_{1} \cdot A_{2}=\frac{r_{e}^{2}-r_{p}^{2}}{5 r^{2}}$ is oblateness coefficient due to smaller primary $m_{2}$, where $r_{e}, r_{p}$ represents equatorial radii and polar radii respectively. $r_{i}(i=1,2)$ are the distance of the infinitesimal mass from $m_{1}$ and $m_{2}$ respectively. Semimajor axis and eccentricity of orbit are denoted by a and e respectively. $\mathrm{c}_{\mathrm{d}}$ is dimensionless velocity of light. For all numerical calculations, we use $\mathrm{a}=0.80, \quad \mathrm{e}=0.20, \quad \mu=0.00003$ and $\mathrm{c}_{\mathrm{d}}=299792458$.

$\mathrm{F}_{\mathrm{x}}, \mathrm{F}_{\mathrm{y}}$ are the partial derivatives of drag force (Kushvah and Ishwar 2006 [4]) with respect to $x$ and $y$ respectively, which are purely functions of the particle's position and velocity. Now multiplying equations (1), (2) and (3) by $2 x^{\prime}, 2 y^{\prime}$ and $2 z^{\prime}$ respectively and adding, we obtain

$\frac{\mathrm{dC}}{\mathrm{dt}}=-2\left(\mathrm{x}^{\prime} \mathrm{F}_{\mathrm{x}}+\mathrm{y}^{\prime} \mathrm{F}_{\mathrm{y}}\right)$

$\mathrm{C}=2 \Omega-\mathrm{x}^{\prime 2}-\mathrm{y}^{\prime 2}$, the quantity $\mathrm{C}$ is Jacobi Integral. The zero velocity curves are given by $\mathrm{C}=2 \Omega$.

\section{Location of triangular equilibrium points}

In order to find the Lagrangian equilibrium points, equations (1), (2) and (3) are solved with the condition that all derivatives are zero, that is

$$
\mathrm{U}_{\mathrm{x}}=\mathrm{U}_{\mathrm{y}}=\mathrm{U}_{\mathrm{z}}=0
$$

$$
\begin{aligned}
& x-\frac{1}{n^{2}}\left\{\frac{(1-\mu)(x+\mu) q_{1}}{r_{1}^{3}}+\frac{\mu(x+\mu-1)}{r_{2}^{3}}+\frac{3 \mu A_{2}(x+\mu-1)}{2 r_{2}^{5}}\right\}-\frac{W_{1}}{n^{2} r_{1}^{2}}\left(\frac{(x+\mu)}{r_{1}^{2}}[(x+\right. \\
& \left.\left.\mu) x^{\prime}+y^{\prime}+z^{\prime}\right]+x^{\prime}-n y\right)=0 \\
& y-\frac{1}{n^{2}}\left\{\frac{(1-\mu) y q_{1}}{r_{1}^{3}}+\frac{\mu y}{r_{2}^{3}}+\frac{3 \mu A_{2} y}{2 r_{2}^{5}}\right\}-\frac{w_{1}}{n^{2} r_{1}^{2}}\left(\frac{y}{r_{1}^{2}}\left[(x+\mu) x^{\prime}+y^{\prime}+z^{\prime}\right]+\right. \\
& \left.y^{\prime}+n(x+\mu)\right)=0 \\
& \left\{\frac{(1-\mu) z q_{1}}{r_{1}^{3}}+\frac{\mu z}{r_{2}^{3}}+\frac{3 \mu A_{2} z}{2 r_{2}^{5}}\right\}+\frac{w_{1}}{r_{1}^{2}}\left(\frac{z}{r_{1}^{2}}\left[(x+\mu) x^{\prime}+y^{\prime}+z^{\prime}\right]+z^{\prime}\right)=0
\end{aligned}
$$

Now, for triangular equilibrium points $\mathrm{U}_{\mathrm{x}}=0, \mathrm{U}_{\mathrm{y}}=0, \mathrm{x} \neq 0, \mathrm{y} \neq$ 0 and $\mathrm{z}=0$ because motion is in $\mathrm{xy}$ plane. Then from equations (8) and (9), we have.

$\mathrm{x}-\frac{1}{\mathrm{n}^{2}}\left\{\frac{(1-\mu)(\mathrm{x}+\mu) \mathrm{q}_{1}}{\mathrm{r}_{1}^{3}}+\frac{\mu(\mathrm{x}+\mu-1)}{\mathrm{r}_{2}^{3}}+\frac{3 \mu \mathrm{A}_{2}(\mathrm{x}+\mu-1)}{2 \mathrm{r}_{2}^{5}}\right\}+\frac{\mathrm{W}_{1}}{\mathrm{n}^{2} \mathrm{r}_{1}^{2}} \mathrm{ny}=0$

$\mathrm{y}-\frac{1}{\mathrm{n}^{2}}\left\{\frac{(1-\mu) \mathrm{yq}_{1}}{\mathrm{r}_{1}^{3}}+\frac{\mu \mathrm{y}}{\mathrm{r}_{2}^{3}}+\frac{3 \mu \mathrm{A}_{2} \mathrm{y}}{2 \mathrm{r}_{2}^{5}}\right\}-\frac{\mathrm{W}_{1}}{\mathrm{n}^{2} \mathrm{r}_{1}^{2}} \mathrm{n}(\mathrm{x}+\mu)=0$

From equation (12), we have

$\left[\mathrm{n}^{2}-\frac{(1-\mu) \mathrm{q}_{1}}{\mathrm{r}_{1}^{3}}-\frac{\mu}{\mathrm{r}_{2}^{3}}-\frac{3 \mu \mathrm{A}_{2}}{2 \mathrm{r}_{2}^{5}}\right] \mathrm{y}_{0}=\frac{\mathrm{w}_{1}}{\mathrm{r}_{1}^{2}} \mathrm{n}(\mathrm{x}+\mu)$

$\mathrm{y}_{0}$ is ordinate of photogravitational ERTBP, which is given by

$\mathrm{y}_{0}= \pm\left[\delta^{2}\left(1-\mathrm{e}^{2}\right)-\frac{1}{4}\left\{1+2\left(\delta^{2}-\mathrm{a}^{\frac{2}{3}}\right)\left(1-\mathrm{e}^{2}\right)\right\}\right]^{\frac{1}{2}}$

where $\delta=\left(\mathrm{aq}_{1}\right)^{\frac{1}{3}}$.

Equations (11) and (12) are multiplied by $y$ and $(x+\mu)$ respectively and subtracting, we have

$\left[\mathrm{n}^{2}-\frac{1}{\mathrm{r}_{2}^{3}}-\frac{3 \mathrm{~A}_{2}}{2 \mathrm{r}_{2}^{5}}\right] \mu \mathrm{y}_{0}=\mathrm{nW}_{1}$.

In photogravitational ERTBP, that is when oblateness and P-R drag is absent and bigger primary is radiating then

$\mathrm{r}_{1}=\left(\frac{\mathrm{q}_{1}}{\mathrm{n}^{2}}\right)^{1 / 3}$

$r_{2}=\frac{1}{n^{2 / 3}}$

Now, we suppose due to P-R drag and oblateness perturbation in $r_{1}$ and $r_{2}$ are $\epsilon_{1}$ and $\epsilon_{2}\left(\epsilon_{1}, \epsilon_{2} \ll 1\right)$ respectively. Then

$r_{1}=\left(\frac{q_{1}}{n^{2}}\right)^{1 / 3}+\epsilon_{1}$
$r_{2}=\frac{1}{n^{2 / 3}}+\epsilon_{2}$

Considering only terms $\mathrm{e}^{2}$ and $\mathrm{A}_{2}$ and neglecting their product, equation (4) gives

$\mathrm{n}^{2}=\frac{1}{\mathrm{a}}\left(1+\frac{3 \mathrm{~A}_{2}}{2}+\frac{3 \mathrm{e}^{2}}{2}\right)$

With the help of equation (19) with $A_{2}=0$, equations (17) and (18) gives

$r_{1}=\left(a_{1}\right)^{1 / 3}\left(1-\frac{e^{2}}{2}\right)+\epsilon_{1}$
$r_{2}=a^{1 / 3}\left(1-\frac{e^{2}}{2}\right)+\epsilon_{2}$

With the help of equations (19), (20) and (21), we have from equations (13) and (14) (taken only first order terms)

$\epsilon_{1}=\frac{\left(\mathrm{aq}_{1}\right)^{1 / 3} \mathrm{a}^{1 / 2} \mathrm{~W}_{1}}{6(1-\mu) \mathrm{y}_{0}}\left(1+\frac{3 \mathrm{~A}_{2}}{4}-\frac{5 \mathrm{e}^{2}}{4}\right)\left[\left\{\left(1+\mathrm{e}^{2}\right)-\mathrm{a}^{2 / 3}\right\}\left(\mathrm{aq}_{1}\right)^{-2 / 3}-\right.$

1] $-\frac{1}{2} \mathrm{~A}_{2}\left(\mathrm{aq}_{1}\right)^{1 / 3}$

$\epsilon_{2}=\frac{\mathrm{W}_{1} \mathrm{a}^{5 / 6}}{3 \mu \mathrm{y}_{0}}\left(1+\frac{3 \mathrm{~A}_{2}}{4}-\frac{5 \mathrm{~A}_{2} \mathrm{a}^{-2 / 3}}{2}-\frac{5 \mathrm{e}^{2}}{4}\right)-\frac{1}{2} \mathrm{~A}_{2} \mathrm{a}^{1 / 3}\left(1-\mathrm{a}^{-2 / 3}\right)(23)$ 
Substituting the values of $\epsilon_{1}$ and $\epsilon_{2}$ in equations (20) and (21) respectively, we have

$\mathrm{r}_{1}=\left(\mathrm{aq}_{1}\right)^{1 / 3}\left(1-\frac{\mathrm{e}^{2}}{2}\right)+\frac{\left(\mathrm{aq}_{1}\right)^{1 / 3} \mathrm{a}^{1 / 2} \mathrm{~W}_{1}}{6(1-\mu) \mathrm{y}_{0}}$

$\left(1+\frac{3 \mathrm{~A}_{2}}{4}-\frac{5 \mathrm{e}^{2}}{4}\right)\left[\left\{\left(1+\mathrm{e}^{2}\right)-\mathrm{a}^{2 / 3}\right\}\left(\mathrm{aq}_{1}\right)^{-2 / 3}-1\right]-$

$\frac{1}{2} \mathrm{~A}_{2}\left(\mathrm{aq}_{1}\right)^{1 / 3}$

$\mathrm{r}_{2}=\mathrm{a}^{1 / 3}\left(1-\frac{\mathrm{e}^{2}}{2}\right)+\frac{\mathrm{W}_{1} \mathrm{a}^{5 / 6}}{3 \mu \mathrm{y}_{0}}\left(1+\frac{3 \mathrm{~A}_{2}}{4}-\frac{5 \mathrm{~A}_{2} \mathrm{a}^{-2 / 3}}{2}-\frac{5 \mathrm{e}^{2}}{4}\right)-$

$\frac{1}{2} A_{2} a^{1 / 3}\left(1-a^{-2 / 3}\right)$

Since, $(x+\mu)=\frac{r_{1}^{2}-r_{2}^{2}+1}{2}, y^{2}=r_{1}^{2}-(x+\mu)^{2}$

Using equations (24),(25) and (26) and solve for $\mathrm{x}, \mathrm{y}$, we have

$\mathrm{x}=\frac{1}{2}-\mu+\frac{1}{2}\left[\left(\mathrm{aq}_{1}\right)^{2 / 3}\left(1-\mathrm{A}_{2}-\mathrm{e}^{2}\right)-\mathrm{a}^{2 / 3}\left(1-\mathrm{A}_{2}-\mathrm{e}^{2}+\right.\right.$

$\left.\mathrm{A}_{2} \mathrm{a}^{-2 / 3}\right]+\frac{\mathrm{W}_{1} \mathrm{a}^{1 / 2}}{3 \mathrm{y}_{0}(1-\mu) \mu}\left\{\left(1+\frac{\mathrm{A}_{2}}{4}-\frac{3 \mathrm{e}^{2}}{4}\right) \frac{\mu}{2}-\left(\mathrm{a}^{2 / 3}+\left(\mathrm{aq}_{1}\right)^{2 / 3}\right)(1+\right.$

$\left.\left.\frac{\mathrm{A}_{2}}{4}-\frac{7 \mathrm{e}^{2}}{4}\right) \frac{\mu}{2}-(1-\mu) \mathrm{a}^{2 / 3}\left(1+\frac{\mathrm{A}_{2}}{4}-2 \mathrm{~A}_{2} \mathrm{a}^{-2 / 3}-\frac{7 \mathrm{e}^{2}}{4}\right)\right\}$ $\mathrm{y}= \pm\left[\left(\mathrm{aq}_{1}\right)^{2 / 3}\left(1-\mathrm{A}_{2}-\mathrm{e}^{2}\right)-\frac{1}{4}\left\{1+2\left(\left(\mathrm{aq}_{1}\right)^{2 / 3}-\mathrm{a}^{2 / 3}\right)(1-\right.\right.$

$\left.\mathrm{e}^{2}\right)+\left(\left(\mathrm{aq}_{1}\right)^{2 / 3}-\mathrm{a}^{2 / 3}\right)^{2}\left(1-2 \mathrm{e}^{2}\right)-2 \mathrm{~A}_{2}\left(1+\left(\mathrm{aq}_{1}\right)^{2 / 3}-\right.$

$\left.\left.\mathrm{a}^{2 / 3}\right)^{2}\right\}+\frac{\mathrm{W}_{1} \mathrm{a}^{1 / 2}}{3 \mathrm{y}_{0}(1-\mu) \mu}\left\{\left(1+\frac{\mathrm{A}_{2}}{4}-\frac{3 \mathrm{e}^{2}}{4}\right) \frac{\mu}{2}-\mu\left(\mathrm{aq}_{1}\right)^{2 / 3}\left(1+\frac{\mathrm{A}_{2}}{4}-\right.\right.$

$\left.\frac{7 \mathrm{e}^{2}}{4}\right)+\left(\left(\mathrm{aq}_{1}\right)^{4 / 3}-\mathrm{a}^{4 / 3}\right)\left(1+\frac{\mathrm{A}_{2}}{4}-\frac{11 \mathrm{e}^{2}}{4}\right) \frac{\mu}{2}+(1-\mu) \mathrm{a}^{2 / 3}(1+$

$\left.\frac{\mathrm{A}_{2}}{4}-2 \mathrm{~A}_{2} \mathrm{a}^{-2 / 3}-\frac{7 \mathrm{e}^{2}}{4}\right)+(1-\mu) \mathrm{a}^{2 / 3}\left(\left(\mathrm{aq}_{1}\right)^{2 / 3}-\mathrm{a}^{2 / 3}\right)\left(1+\frac{\mathrm{A}_{2}}{4}-\right.$

$\left.2 \mathrm{~A}_{2} \mathrm{a}^{-2 / 3}-\frac{11 \mathrm{e}^{2}}{4}\right)-\frac{\mu}{2} \mathrm{~A}_{2}\left(1+\left(\mathrm{aq}_{1}\right)^{2 / 3}-\mathrm{a}^{2 / 3}\right)\left(\left(\mathrm{aq}_{1}\right)^{2 / 3}+\right.$

$\left.\left.\left.a^{2 / 3}-1\right)-(1-\mu) a^{2 / 3}\left(1+\left(a q_{1}\right)^{2 / 3}-a^{2 / 3}\right) A_{2}\right\}\right]^{1 / 2}$

The position of triangular equilibrium points $\left(\mathrm{L}_{4}(5)\right)$ is given by equations (27) and (28) which are valid for $W_{1} \ll 1, A_{2} \ll 1$. Figure 1 shows that perturbations $\left(\epsilon_{1}, \epsilon_{2}\right)$ in $r_{1}$ and $r_{2}$ will become zero, when $A_{2}=0$ and $q_{1}=1$. From figure 2 , it is clear that $x, y$ are increasing function of $q_{1}$ and decreasing function of $A_{2}$. Numerical values of coordinate $(\mathrm{x}, \mathrm{y})$ of triangular equilibrium points $\left(\mathrm{L}_{4(5)}\right)$ are given in table 1 and table 2, for different values of $\mathrm{A}_{2}$ and $\mathrm{q}_{1}$. For the numerical calculations we have taken $\mu$ $=0.00003$, e=0.2, a =0.8, $c_{d}=299792458,0 \leq \mathrm{q}_{1} \leq 1,0 \leq \mathrm{A}_{2} \leq 1$.
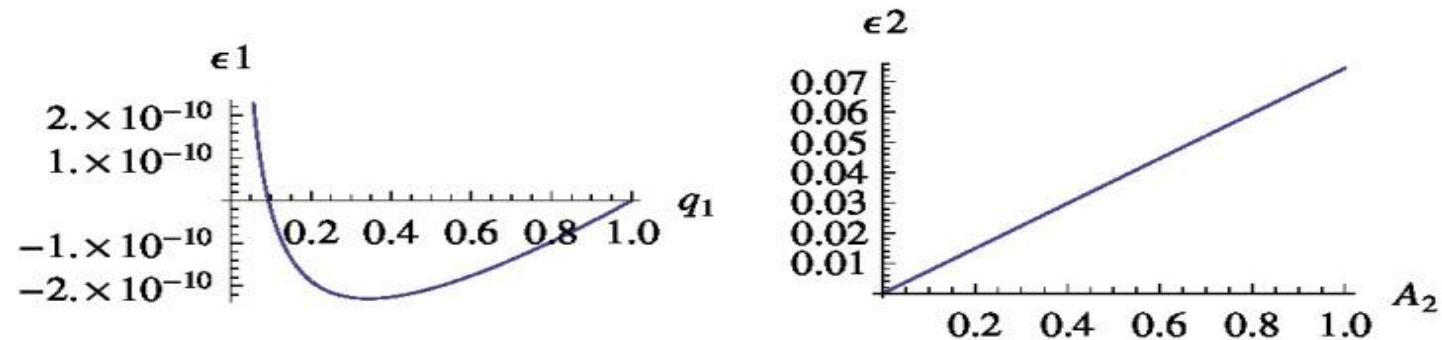

Fig. 1: The effect of $A_{2}$ and $q_{1}$ on $\epsilon_{1}$ and $\epsilon_{2}$. when $e=0.2, a=0.8, \mu=0.00003, c_{d}=299792458,0 \leq q_{1} \leq 1,0 \leq A_{2} \leq 1$
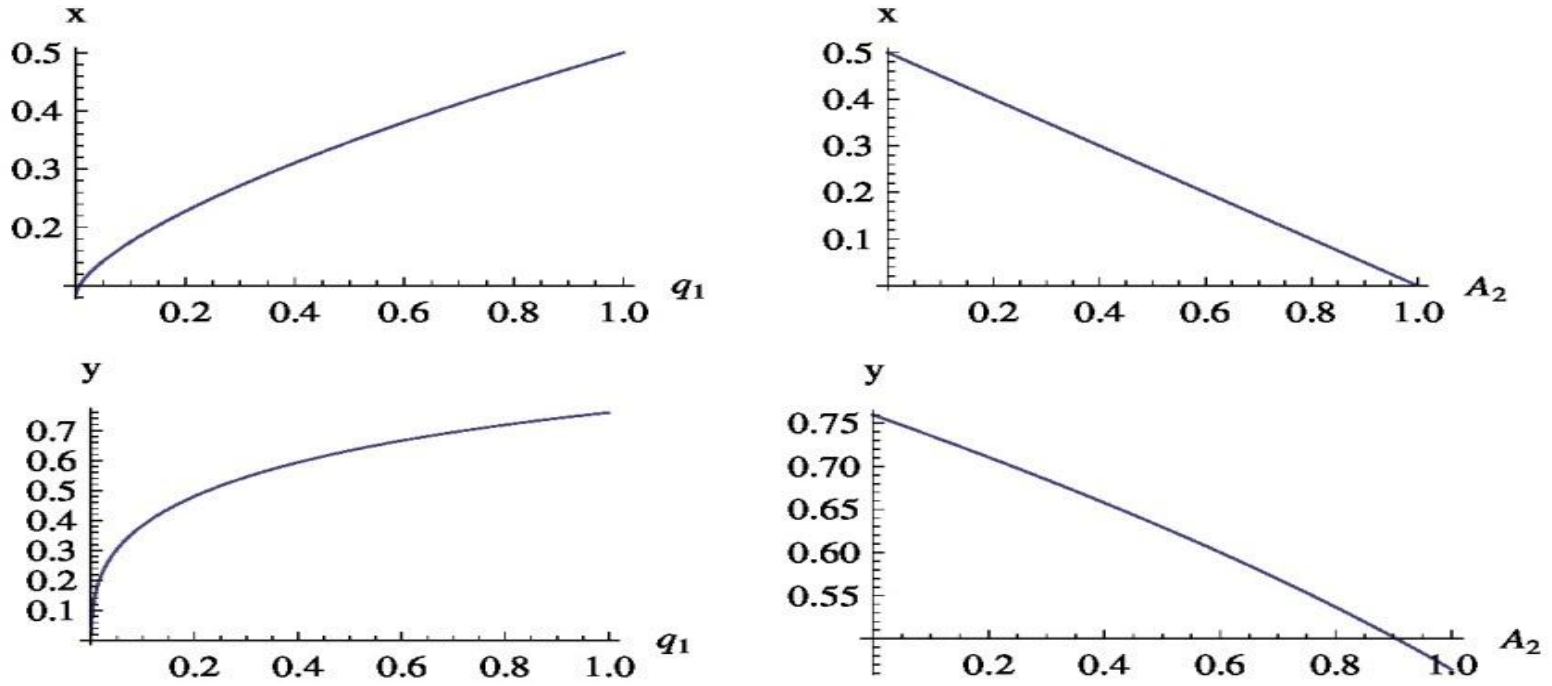

Fig. 2: The effect of $A_{2}$ and $\mathrm{q}_{1}$ on $\mathrm{x}$, y co-ordinates of triangular equilibrium points $\mathrm{L}_{4(5)}$ ). when $\mathrm{e}=0.2, \mathrm{a}=0.8, \mu=0.00003, \mathrm{c}_{\mathrm{d}}=299792458,0 \leq$ $\mathrm{q}_{1} \leq 1,0 \leq \mathrm{A}_{2} \leq 1$.

Table 1: $x$ co-ordinate of $\mathrm{L}_{4}$

\begin{tabular}{llllll}
\hline $\mathrm{A}_{2}$ & \multicolumn{1}{c}{$\mathrm{x}_{4}: \mathrm{q}_{1}=1$} & \multicolumn{1}{c}{$\mathrm{x}_{4}: \mathrm{q}_{1}=0.75$} & \multicolumn{1}{c}{$\mathrm{x}_{4}: \mathrm{q}_{1}=0.50$} & \multicolumn{1}{c}{$\mathrm{x}_{4}: \mathrm{q}_{1}=0.25$} \\
\hline 0.00 & 0.49997 & 0.427771 & 0.346882 & 0.250438 & Complex Number \\
0.25 & 0.37497 & 0.321576 & 0.261755 & 0.190431 & Complex Number \\
0.50 & 0.24997 & 0.21538 & 0.176628 & 0.130425 & Complex Number \\
0.75 & 0.12497 & 0.109185 & 0.0915006 & 0.0704191 & Complex Number \\
1.00 & -0.00003 & 0.00298943 & 0.00637354 & 0.010413 & Complex Number \\
\hline
\end{tabular}

Notes: Numerical values of $x$ coordinate of $L_{4}$ for different values of $A_{2}$ and $q_{1} .0 \leq A_{2}, q_{1} \leq 1$. 
Table 2: y co-ordinate of $\mathrm{L}_{4}$

\begin{tabular}{|c|c|c|c|c|c|}
\hline 2 & $\mathrm{y}_{4}: \mathrm{q}_{1}=1$ & $\mathrm{y}_{4}: \mathrm{q}_{1}=0.75$ & $\mathrm{y}_{4}: \mathrm{q}_{1}=0.50$ & $\mathrm{y}_{4}: \mathrm{q}_{1}=0.25$ & $\mathrm{y}_{4}: \mathrm{q}_{1}=0$ \\
\hline 0.00 & 0.759805 & 0.707049 & 0.633136 & 0.515451 & Complex Number \\
\hline 0.25 & 0.697753 & 0.642103 & 0.568434 & 0.457167 & Complex Number \\
\hline 0.50 & 0.629616 & 0.59801 & 0.495352 & 0.390275 & Complex Number \\
\hline 0.75 & 0.553148 & 0.486879 & 0.409426 & 0.309236 & Complex Number \\
\hline 1.00 & 0.464251 & 0.386559 & 0.299809 & 0.197330 & Complex Number \\
\hline
\end{tabular}

Notes: Numerical values of y coordinate of $L_{4}$ for different values of $A_{2}$ and $q_{1} .0 \leq A_{2}, q_{1} \leq 1$

\section{Stability of triangular equilibrium points}

Rewriting equations of motion, we have

$\mathrm{x}^{\prime \prime}-2 \mathrm{y}^{\prime}=\frac{\partial \Omega}{\partial \mathrm{x}}-\frac{\mathrm{W}_{1} \mathrm{~N}_{1}}{\mathrm{n}^{2} \mathrm{r}_{1}^{2} \sqrt{1-\mathrm{e}^{2}}}=\mathrm{U}_{\mathrm{x}}$

$\mathrm{y}^{\prime \prime}+2 \mathrm{x}^{\prime}=\frac{\partial \Omega}{\partial \mathrm{y}}-\frac{\mathrm{W}_{1} \mathrm{~N}_{2}}{\mathrm{n}^{2} \mathrm{r}_{1}^{2} \sqrt{1-\mathrm{e}^{2}}}=\mathrm{U}_{\mathrm{y}}$

$\mathrm{z}^{\prime \prime}=\frac{\partial \Omega}{\partial \mathrm{z}}-\frac{\mathrm{W}_{1} \mathrm{~N}_{3}}{\mathrm{n}^{2} \mathrm{r}_{1}^{2} \sqrt{1-\mathrm{e}^{2}}}=\mathrm{U}_{\mathrm{z}}$

where,

$\mathrm{N}_{1}=\frac{(\mathrm{x}+\mu) \mathrm{N}}{\mathrm{r}_{1}^{2}}+\mathrm{x}^{\prime}-\mathrm{ny}$,

$\mathrm{N}_{2}=\frac{\mathrm{yN}}{\mathrm{r}_{1}^{2}}+\mathrm{y}^{\prime}+\mathrm{n}(\mathrm{x}+\mu)$

$\mathrm{N}_{3}=\frac{\mathrm{zN}}{\mathrm{r}_{1}^{2}}+\mathrm{z}^{\prime}, \mathrm{N}=(\mathrm{x}+\mu) \mathrm{x}^{\prime}+\mathrm{yy}^{\prime}+\mathrm{zz}^{\prime}$.

We suppose that $\alpha, \beta, \gamma$ be the small displacement from equilibrium points $\left(\mathrm{x}_{*}, \mathrm{y}_{*}, \mathrm{z}_{*}\right)$ then $\mathrm{x}=\mathrm{x}_{*}+\alpha, \mathrm{y}=\mathrm{y}_{*}+\beta, \mathrm{z}=\mathrm{z}_{*}+\gamma$. At equilibrium points $\left(\mathrm{x}_{*}, \mathrm{y}_{*}, \mathrm{z}_{*}\right), \mathrm{x}^{\prime}{ }_{*}=\mathrm{y}^{\prime}{ }_{*}=\mathrm{z}^{\prime}{ }_{*}=\mathrm{x}^{\prime \prime}{ }_{*}=\mathrm{y}^{\prime \prime}=\mathrm{z}^{\prime \prime}{ }_{*}=$ 0 . Hence, the equations of motion corresponding to the system of equations (29), (30) and (31), by using Taylor's theorem, are written as

$\alpha^{\prime \prime}-2 \beta^{\prime}=\mathrm{U}_{\mathrm{x}}^{0}+\alpha \mathrm{U}_{\mathrm{xx}}^{0}+\beta \mathrm{U}_{\mathrm{xy}}^{0}+\gamma \mathrm{U}_{\mathrm{xz}}^{0}+\alpha^{\prime} \mathrm{U}_{\mathrm{xx}}^{0}+\beta^{\prime} \mathrm{U}_{\mathrm{xy}}^{0}+\gamma^{\prime} \mathrm{U}_{\mathrm{xz}}^{0}$

$\beta^{\prime \prime}+2 \alpha^{\prime}=\mathrm{U}_{\mathrm{y}}^{0}+\alpha \mathrm{U}_{\mathrm{yx}}^{0}+\beta \mathrm{U}_{\mathrm{yy}}^{0}+\gamma \mathrm{U}_{\mathrm{yz}}^{0}+\alpha^{\prime} \mathrm{U}_{\mathrm{yx}^{\prime}}^{0}+\beta^{\prime} \mathrm{U}_{\mathrm{yy}^{\prime}}^{0}+\gamma^{\prime} \mathrm{U}_{\mathrm{yz}}^{0}$

$\gamma^{\prime \prime}=\mathrm{U}_{\mathrm{z}}^{0}+\alpha \mathrm{U}_{\mathrm{zx}}^{0}+\beta \mathrm{U}_{\mathrm{zy}}^{0}+\gamma \mathrm{U}_{\mathrm{zz}}^{0}+\alpha^{\prime} \mathrm{U}_{\mathrm{zx}^{\prime}}^{0}+\beta^{\prime} \mathrm{U}_{\mathrm{zy}^{\prime}}^{0}+\gamma^{\prime} \mathrm{U}_{\mathrm{zz}^{\prime}}^{0}$

where superscript ' 0 ' indicates that the partial derivatives are to be evaluated at the equilibrium point $\left(\mathrm{x}_{*}, \mathrm{y}_{*}, \mathrm{z}_{*}\right)$. At $\left(\mathrm{x}_{*}, \mathrm{y}_{*}, \mathrm{z}_{*}, \mathrm{U}_{\mathrm{x}}^{0}=\right.$ $\mathrm{U}_{\mathrm{y}}^{0}=\mathrm{U}_{\mathrm{z}}^{0}=\mathrm{U}_{\mathrm{xz}}^{0}=\mathrm{U}_{\mathrm{yz}}^{0}=\mathrm{U}_{\mathrm{zx}}^{0}=\mathrm{U}_{\mathrm{zy}}^{0}=\mathrm{U}_{\mathrm{xz}^{\prime}}^{0}=\mathrm{U}_{\mathrm{yz}^{\prime}}^{0}=\mathrm{U}_{\mathrm{zx}^{\prime}}^{0}=$

$\mathrm{U}_{\mathrm{yz}^{\prime}}^{0}=0$. Hence system of equations (32), (33) and (34) are written as

$\alpha^{\prime \prime}-2 \beta^{\prime}=\alpha \mathrm{U}_{\mathrm{xx}}^{0}+\beta \mathrm{U}_{\mathrm{xy}}^{0}+\alpha^{\prime} \mathrm{U}_{\mathrm{xx}^{\prime}}^{0}+\beta^{\prime} \mathrm{U}_{\mathrm{xy}^{\prime}}^{0}$

$\beta^{\prime \prime}+2 \alpha^{\prime}=\alpha \mathrm{U}_{\mathrm{yx}}^{0}+\beta \mathrm{U}_{\mathrm{yy}}^{0}+\alpha^{\prime} \mathrm{U}_{\mathrm{yx}^{\prime}}^{0}+\beta^{\prime} \mathrm{U}_{\mathrm{yy}}^{0}$

$\gamma^{\prime \prime}=\gamma \mathrm{U}_{\mathrm{zz}}^{0}+\gamma^{\prime} \mathrm{U}_{\mathrm{zz}}^{0}$.

The value of second order partial derivatives at $\left(\mathrm{x}_{*,} \mathrm{y}_{*}, 0\right)$ are

$\mathrm{U}_{\mathrm{xx}}^{0}=$

$\frac{1}{\sqrt{1-\mathrm{e}^{2}}}\left[1-\mathrm{a}\left(1-\frac{3 \mathrm{~A}_{2}}{2}-\frac{3 \mathrm{e}^{2}}{2}\right)\left(\mathrm{f}_{*}-3 \mathrm{~J}_{1 *}\right)-\frac{2 \mathrm{~W}_{1} \mathrm{a}^{1 / 2}\left(\mathrm{x}_{*},+\mu\right) \mathrm{y}_{*}}{\mathrm{r}_{1 *}^{4}}(1-\right.$

$\left.\left.\frac{3 \mathrm{~A}_{2}}{4}-\frac{3 \mathrm{e}^{2}}{4}\right)\right]$

$\mathrm{U}_{\mathrm{xy}}^{0}=\frac{\mathrm{a}}{\sqrt{1-\mathrm{e}^{2}}}\left[3 \mathrm{~g}_{*}\left(1-\frac{3 \mathrm{~A}_{2}}{2}-\frac{3 \mathrm{e}^{2}}{2}\right)+\frac{\mathrm{w}_{1} \mathrm{a}^{-1 / 2}}{\mathrm{r}_{1 *}^{2}}\left(1-\frac{3 \mathrm{~A}_{2}}{4}-\frac{3 \mathrm{e}^{2}}{4}\right)(1-\right.$

$\left.\left.\frac{2 \mathrm{y}_{*}^{2}}{\mathrm{r}_{1 *}^{2}}\right)\right]$

$\mathrm{U}_{\mathrm{xx}^{\prime}}^{0}=-\frac{\mathrm{W}_{1} \mathrm{a}}{\mathrm{r}_{1 *}^{2} \sqrt{1-\mathrm{e}^{2}}}\left(1-\frac{3 \mathrm{~A}_{2}}{2}-\frac{3 \mathrm{e}^{2}}{2}\right)\left[1+\frac{\left(\mathrm{x}_{*}+\mu\right)^{2}}{\mathrm{r}_{1 *}^{2}}\right]$

$\mathrm{U}_{\mathrm{yx}}^{0}=\frac{\mathrm{a}}{\sqrt{1-\mathrm{e}^{2}}}\left[3 \mathrm{~g}_{*}\left(1-\frac{3 \mathrm{~A}_{2}}{2}-\frac{3 \mathrm{e}^{2}}{2}\right)-\frac{\mathrm{w}_{1} \mathrm{a}^{-1 / 2}}{\mathrm{r}_{1 *}^{2}}\left(1-\frac{3 \mathrm{~A}_{2}}{4}-\frac{3 \mathrm{e}^{2}}{4}\right)(1-\right.$ $\left.\left.\frac{2\left(\mathrm{x}_{*}, \mu\right)^{2}}{\mathrm{r}_{1 *}^{2}}\right)\right]$
$\mathrm{U}_{\mathrm{yy}}^{0}=$

$\frac{1}{\sqrt{1-\mathrm{e}^{2}}}\left[1-\mathrm{a}\left(1-\frac{3 \mathrm{~A}_{2}}{2}-\frac{3 \mathrm{e}^{2}}{2}\right)\left(\mathrm{f}_{*}-3 \mathrm{~J}_{2 *}\right)+\frac{2 \mathrm{~W}_{1} \mathrm{a}^{1 / 2}\left(\mathrm{x}_{*}, \mu\right) \mathrm{y}_{*}}{\mathrm{r}_{1 *}^{4}}\left(1-\frac{3 \mathrm{~A}_{2}}{4}-\right.\right.$

$\left.\left.\frac{3 \mathrm{e}^{2}}{4}\right)\right]$

$\mathrm{U}_{\mathrm{yy}}^{0}=-\frac{\mathrm{W}_{1} \mathrm{a}}{\mathrm{r}_{1 *}^{2} \sqrt{1-\mathrm{e}^{2}}}\left(1-\frac{3 \mathrm{~A}_{2}}{2}-\frac{3 \mathrm{e}^{2}}{2}\right)\left[1+\frac{\mathrm{y}_{*}^{2}}{\mathrm{r}_{2 *}^{1}}\right]$

$\mathrm{U}_{\mathrm{xy}^{\prime}}^{0}=-\frac{\mathrm{W}_{1} \mathrm{a}}{\mathrm{r}_{1 *}^{4} \sqrt{1-\mathrm{e}^{2}}}\left(1-\frac{3 \mathrm{~A}_{2}}{2}-\frac{3 \mathrm{e}^{2}}{2}\right)\left(\mathrm{x}_{*}+\mu\right) \mathrm{y}_{*}=\mathrm{U}_{\mathrm{yx}^{\prime}}^{0}$

$\mathrm{U}_{\mathrm{zZ}}^{0}=\frac{-\mathrm{a}}{\sqrt{1-\mathrm{e}^{2}}}\left(1-\frac{3 \mathrm{~A}_{2}}{2}-\frac{3 \mathrm{e}^{2}}{2}\right)\left[\frac{(1-\mu) \mathrm{q}_{1}}{\mathrm{r}_{1 *}^{3}}+\frac{\mu}{\mathrm{r}_{2 *}^{3}}+\frac{3 \mu \mathrm{A}_{2}}{2 \mathrm{r}_{2 *}^{5}}\right]$

$\mathrm{U}_{\mathrm{zz}}^{0}=-\frac{\mathrm{W}_{1} \mathrm{a}}{\mathrm{r}_{1 *}^{2} \sqrt{1-\mathrm{e}^{2}}}\left(1-\frac{3 \mathrm{~A}_{2}}{2}-\frac{3 \mathrm{e}^{2}}{2}\right)$

where

$\mathrm{f}_{*}=\frac{(1-\mu) \mathrm{q}_{1}}{\mathrm{r}_{1 *}^{3}}+\frac{\mu}{\mathrm{r}_{2 *}^{3}}+\frac{3 \mu \mathrm{A}_{2}}{2 \mathrm{r}_{2 *}^{5}}$

$\mathrm{g}_{*}=\frac{\mathrm{q}_{1}(1-\mu)\left(\mathrm{x}_{*}+\mu\right) \mathrm{y}_{*}}{\mathrm{r}_{1 *}^{5}}+\frac{\mu\left(\mathrm{x}_{*}+\mu-1\right) \mathrm{y}_{*}}{\mathrm{r}_{2 *}^{5}}+\frac{5 \mu \mathrm{A}_{2}\left(\mathrm{x}_{*}+\mu-1\right) \mathrm{y}_{*}}{2 \mathrm{r}_{2 *}^{7}}$

$\mathrm{J}_{1 *}=\frac{\mathrm{q}_{1}(1-\mu)\left(\mathrm{x}_{*}+\mu\right)^{2}}{\mathrm{r}_{1 *}^{5}}+\frac{\mu\left(\mathrm{x}_{*}+\mu-1\right)^{2}}{\mathrm{r}_{2 *}^{5}}+\frac{5 \mu \mathrm{A}_{2}\left(\mathrm{x}_{*}+\mu-1\right)^{2}}{2 \mathrm{r}_{2 *}^{7}}$

$\mathrm{J}_{2 *}=\frac{\mathrm{q}_{1}(1-\mu) \mathrm{y}_{*}{ }^{2}}{\mathrm{r}_{1 *}^{5}}+\frac{\mu \mathrm{y}_{*}{ }^{2}}{\mathrm{r}_{2 *}^{5}}+\frac{5 \mu \mathrm{A}_{2} \mathrm{y}_{*}{ }^{2}}{2 \mathrm{r}_{2 *}^{7}}$

Star $(*)$ represents the value at equilibrium point $\left(\mathrm{x}_{*} \mathrm{y}_{*}, \mathrm{z}_{*}\right)$. We suppose $\alpha=\mathrm{A}_{1} \mathrm{e}^{\lambda \mathrm{t}}, \beta=\mathrm{B}_{1} \mathrm{e}^{\lambda \mathrm{t}}$ and $\gamma=\mathrm{C}_{1} \mathrm{e}^{\lambda \mathrm{t}}$. Using these values, the system of equations (35), (36) and (37) are written as

$\left(\lambda^{2}-\mathrm{U}_{\mathrm{xx}}^{0}-\lambda \mathrm{U}_{\mathrm{xx}}^{0}\right) \mathrm{A}_{1}+\left[-\left(2+\mathrm{U}_{\mathrm{xy}}^{0}\right) \lambda-\mathrm{U}_{\mathrm{xy}}^{0}\right] \mathrm{B}_{1}=0$

$\left[\left(2-\mathrm{U}_{\mathrm{yx}}^{0}\right) \lambda-\mathrm{U}_{\mathrm{yx}}^{0}\right] \mathrm{A}_{1}+\left(\lambda^{2}-\lambda \mathrm{U}_{\mathrm{yy}}^{0}-\mathrm{U}_{\mathrm{yy}}^{0}\right) \mathrm{B}_{1}=0$

$\left(\lambda^{2}-\lambda \mathrm{U}_{\mathrm{zz}}^{0}-\mathrm{U}_{\mathrm{zz}}^{0}\right) \mathrm{C}_{1}=0$

From equation (40), we have $\lambda=\frac{\mathrm{U}_{\mathrm{zz}}^{0} \pm \sqrt{\left(\mathrm{U}_{\mathrm{zz}}^{0}\right)^{2}+4 \mathrm{U}_{\mathrm{zz}}^{0}}}{2}$. After substituting values of partial derivatives, we have

$\lambda=$

$\frac{\mathrm{a}}{2}\left(1-\frac{3 \mathrm{~A}_{2}}{2}-\frac{3 \mathrm{e}^{2}}{2}\right)\left[\frac{-\mathrm{W}_{1}}{\mathrm{r}_{1 *}^{2} \sqrt{1-\mathrm{e}^{2}}} \pm \sqrt{\frac{\mathrm{W}_{1}^{2}}{\mathrm{r}_{1 *}^{4} \sqrt{1-\mathrm{e}^{2}}}-\frac{4 \mathrm{f}_{*}}{\mathrm{a} \sqrt{1-\mathrm{e}^{2}}}\left(1+\frac{3 \mathrm{~A}_{2}}{2}+\frac{3 \mathrm{e}^{2}}{2}\right)}\right]$

Since $\lambda$ consists always negative real part, hence motion is asymptotically stable in $\mathrm{z}$ direction. Now equations (38) and (39) have singular solution if

$$
\left|\begin{array}{cc}
\lambda^{2}-\mathrm{U}_{\mathrm{xx}}^{0}-\lambda \mathrm{U}_{\mathrm{xx}}^{0} & -\left(2+\mathrm{U}_{\mathrm{xy}}^{0}\right) \lambda-\mathrm{U}_{\mathrm{xy}}^{0} \\
\left(2-\mathrm{U}_{\mathrm{yx}}^{0}\right) \lambda-\mathrm{U}_{\mathrm{yx}}^{0} & \lambda^{2}-\lambda \mathrm{U}_{\mathrm{yy}^{\prime}}^{0}-\mathrm{U}_{\mathrm{yy}}^{0}
\end{array}\right|=0
$$

ie

$\lambda^{4}+a_{0} \lambda^{3}+a_{1} \lambda^{2}+a_{2} \lambda+a_{3}=0$

This is the characteristic equation of the problem. $a_{0}, a_{1}, a_{2}$ and $a_{3}$ are coefficients, where

$a_{0}=-\left(U_{x x^{\prime}}^{0}+U_{y y^{\prime}}^{0}\right)$

$a_{1}=4-\left(U_{x x}^{0}+U_{y y}^{0}\right)+2\left(U_{x y^{\prime}}^{0}-U_{y x^{\prime}}^{0}\right)+U_{x x^{\prime}}^{0} U_{y y^{\prime}}^{0}-$

$U_{x y^{\prime}}^{0} U_{y x^{\prime}}^{0}$ 
$a_{2}=U_{x x}^{0} U_{y y^{\prime}}^{0}+U_{x x^{\prime}}^{0} U_{y y}^{0}+2\left(U_{x y}^{0}-U_{y x}^{0}\right)-U_{x y^{\prime}}^{0} U_{y x}^{0}-$

$U_{x y}^{0} U_{y x^{\prime}}^{0}$

$a_{3}=U_{x x}^{0} U_{y y}^{0}-U_{x y}^{0} U_{y x}^{0}$.

At equilibrium point, we get

$a_{0}=\frac{3 W_{1} \mathrm{a}}{r_{1 *}^{2} \sqrt{1-e^{2}}}\left(1-\frac{3 A_{2}}{2}-\frac{3 e^{2}}{2}\right)$

$a_{1}=4-\frac{\mathrm{a}}{\sqrt{1-e^{2}}}\left(1-\frac{3 A_{2}}{2}-\frac{3 e^{2}}{2}\right)\left[2+f_{*}+\frac{3 \mu A_{2}}{r_{2 *}^{5}}\right]$

$+\frac{2 W_{1}^{2} \mathrm{a}}{\left(1-e^{2}\right) r_{1 *}^{4}}\left(1-\frac{3 A_{2}}{2}-\frac{3 e^{2}}{2}\right)=b_{0}+b_{1}$

$b_{0}=4-\frac{\mathrm{a}}{\sqrt{1-e^{2}}}\left(1-\frac{3 A_{2}}{2}-\frac{3 e^{2}}{2}\right)\left[2+f_{*}+\frac{3 \mu A_{2}}{r_{2 *}^{5}}\right]$

$b_{1}=\frac{2 W_{1}^{2} \mathrm{a}}{\left(1-e^{2}\right) r_{1 *}^{4}}\left(1-\frac{3 A_{2}}{2}-\frac{3 e^{2}}{2}\right)$

$a_{2}=\frac{-a_{1}}{\sqrt{1-e^{2}}}\left[1+\mathrm{a}\left(1-\frac{3 A_{2}}{2}-\frac{3 e^{2}}{2}\right)\left\{\frac{\mu A_{2}}{r_{2 *}^{5}}+\frac{\mu}{r_{1 *}^{2} r_{2 *}^{5}}\left(1+\frac{5 A_{2}}{2 r_{2 *}^{2}}\right) y_{*}^{2}\right\}\right]$

$a_{3}=\frac{1}{1-e^{2}}\left[\left\{1-\mathrm{a} f_{*}\left(1-\frac{3 A_{2}}{2}-\frac{3 e^{2}}{2}\right)\right\}\left\{1+\mathrm{a}\left(1-\frac{3 A_{2}}{2}-\right.\right.\right.$

$\left.\left.\frac{3 e^{2}}{2}\right)\left(2 f_{*}+\frac{3 \mu A_{2}}{r_{2 *}^{5}}\right)\right\}+\frac{9 \mathrm{a}^{2}\left(1-3 A_{2}-3 e^{2}\right) \mu(1-\mu) q_{1}}{r_{1 *}^{5} r_{2 *}^{5}}\left(1+\frac{5 A_{2}}{2 r_{2 *}^{2}}\right) y_{*}^{2}+$

$\frac{6 W_{1} \mathrm{a}^{3 / 2}}{r_{1 *}^{4}}\left(1-\frac{9 A_{2}}{4}-\frac{9 e^{2}}{4}\right)\left\{\frac{-\mu y_{*}}{r_{2 *}^{5}}\left(1+\frac{5 A_{2}}{2 r_{2 *}^{2}}\right)\left(\left(x_{*}+\mu\right)^{2}+y_{*}^{2}-\right.\right.$

$\left.\left.\left(x_{*}+\mu\right)\right\}-\frac{W_{1}^{2} \mathrm{a}}{r_{1 *}^{4}}\left(1-\frac{3 A_{2}}{2}-\frac{3 e^{2}}{2}\right)\right]$.

We write the four roots of the classical characteristic equation as

$\lambda_{j}= \pm z i$ where $\mathrm{j}=1,2,3,4$

$z^{2}=\frac{1}{2}\left\{1 \mp[1-27 \mu(1-\mu)]^{1 / 2}\right\}$.

Then

$\lambda_{1,2}= \pm\left(\frac{27}{4} \mu(1-\mu)\right)^{1 / 2} i$

$\lambda_{3,4}= \pm\left(1-\frac{27}{4} \mu(1-\mu)\right)^{1 / 2} i$

$\lambda_{1,2}, \lambda_{3,4}$ represent the four roots of classical case where $i=\sqrt{-1}$. Due to P-R drag and oblateness, we suppose the solution of the characteristic equation (38) is of the form $\lambda=\lambda_{j}\left(1+e_{1}+e_{2} i\right)=$ $\pm\left[-e_{2}+\left(1+e_{1}\right) i\right] z$, where $e_{1}, e_{2}$ are small. We have in first order approximation

$\lambda^{2}=\left[-e_{2}+\left(1+e_{1}\right) i\right]^{2} z^{2}$

$\lambda^{2}=\left[-\left(1+2 e_{1}\right)-2 e_{2} i\right] z^{2}$

$\lambda^{3}= \pm\left[3 e_{2}-\left(1+3 e_{1}\right) i\right] z^{3}$

$\lambda^{4}=\left[1+4 e_{1}+4 e_{2} i\right] z^{4}$.

Substituting these values in equation (41), neglecting product of $e_{1}, e_{2}$ with $a_{0}, a_{1}, a_{2}$, and $a_{3}$, we get

$e_{1}=\frac{-a_{3}+b_{0} z^{2}-z^{4}}{2 z^{2}\left(2 z^{2}-b_{0}\right)}$

$e_{2}=\frac{\mp a_{2} z \pm a_{0} z^{3}}{2 z^{2}\left(2 z^{2}-b_{0}\right)}$

If $e_{2} \neq 0$, then the resulting motion of particle displaced from equilibrium points is asymptotically stable only when all the real part of $\lambda$ are negative. For stability, we require $\operatorname{Re}(\lambda)<0$, $\operatorname{Re}(\lambda)=\frac{a_{2}-a_{0} z^{2}}{2\left(2 z^{2}-1\right)}$. Taking positive sign from equation (46), we have $z^{2}=1-\frac{27}{4} \mu(1-\mu)$

We consider $\operatorname{Re}(\lambda)<0$, then

$\left\{a_{2}-a_{0}\left(1-\frac{27}{4} \mu(1-\mu)\right)\right\}\left\{1-\frac{27}{2} \mu(1-\mu)\right\}^{-1}<0$

$a_{2}+\frac{27}{4} \mu(1-\mu)\left(2 a_{2}-a_{0}\right)<a_{0}$

Taking negative sign from equation (46), we have

$0<a_{2}+\frac{27}{4} \mu(1-\mu)\left(2 a_{2}-a_{0}\right)$

From equations (55) and (56), we get

$0<a_{2}+\frac{27}{4} \mu(1-\mu)\left(2 a_{2}-a_{0}\right)<a_{0}$

$0<a_{2}<a_{0}$ as $\mu \rightarrow 0$ Murray [6]

Inequality (57) is necessary condition for stability of triangular equilibrium points $L_{4(5)}$. When $W_{1} \neq 0, A_{2} \neq 0$ and $n^{2}=$ $\frac{1}{a}\left(1+\frac{3 A_{2}}{2}+\frac{3 e^{2}}{2}\right)$

$a_{0}=\frac{3 W_{1} \mathrm{a}}{\mathrm{r}_{1 *}^{2} \sqrt{1-\mathrm{e}^{2}}}\left(1-\frac{3 \mathrm{~A}_{2}}{2}-\frac{3 \mathrm{e}^{2}}{2}\right)$

$a_{2}=\frac{-a_{1}}{\sqrt{1-\mathrm{e}^{2}}}\left[1+\mathrm{a}\left(1-\frac{3 \mathrm{~A}_{2}}{2}-\frac{3 \mathrm{e}^{2}}{2}\right)\left\{\frac{\mu \mathrm{A}_{2}}{\mathrm{r}_{2 *}^{5}}+\frac{\mu}{\mathrm{r}_{1 *}^{2} \mathrm{r}_{2 *}^{5}}\left(1+\frac{5 \mathrm{~A}_{2}}{2 \mathrm{r}_{2 *}^{2}}\right)\right\}\right](59)$

Since $\mathrm{W}_{1}>0$ hence $a_{0}>0$. From equation (59) it is clear $a_{2}$ is always negative that is $a_{2}<0$. If we consider P-R drag and oblateness then $a_{2}<0$. This does not satisfy the necessary condition of stability, hence motion is unstable in linear sense.

\section{Conclusion}

We have studied the effect of P-R drag, radiation and oblateness on triangular equilibrium points in elliptic restricted three body problem. We have found that locations of triangular points are different from classical case. If we put $\mathrm{W}_{1}=0, \mathrm{~A}_{2}=0, \mathrm{q}_{1}=$ $1, \mathrm{a}=1$ and $\mathrm{e}=0$ in equations (27) and (28), then we get $\mathrm{x}=\frac{1}{2}-$ $\mu, \mathrm{y}= \pm \frac{\sqrt{3}}{2}$. These are coordinate of classical RTBP. In this case $27 \mu(1-\mu)<1$, that is $\mu<0.03852089$. If we do not consider P-R drag $\left(\mathrm{W}_{1}=0\right)$ then $\mathrm{L}_{4(5)}$ coincide with Singh and Umar [12]. With $\mathrm{a}=1, \mathrm{e}=0$ and $\mathrm{A}_{2}=0$ triangular equilibrium points are same as Schuerman [10]. Kushvah [4] studied same problem in circular case. He found that triangular equilibrium points are unstable in his problem. When we put $\mathrm{a}=1$ and $\mathrm{e}=0$ in equation (27) and (28), triangular equilibrium points are similar as in Kushvah [4]. From figure 2, we find that $\mathrm{x}$ and $\mathrm{y}$ both are increasing function of $q_{1}$ and decreasing function of $A_{2}$. Numerical values of $\mathrm{x}$ and $\mathrm{y}$ are given in table 1 and table 2 . At last with the help of Murray [6], we conclude that triangular equilibrium points are unstable in linear sense in our problem.

\section{Acknowledgement}

We are thankful to D.S.T. Government of India, New Delhi for sanctioning the project SR/S4/MS: 728/11, dated:15/06/2013 on this topic and IUCAA, Pune for providing local hospitality and facility of library and computer centre for research work.

\section{References}

[1] Broucke,R.,(1969): Stability of periodic orbits in the elliptic restricted three body problem, AIAA Journal, 7(6), 1003-1009 http://dx.doi.org/10.2514/3.5267. 
[2] Chernikov Yu. A., (1970): The photogravitational restricted three body problems, Soviet Astronomy-AJ, 14(1), 176-181.

[3] Danby,J.M.A.,(1964): Stability of the triangular points in the elliptic restricted problem of three bodies, The Astronomical Journal,69(2), 165-172.http://dx.doi.org/10.1086/109254.

[4] Kushvah,B.S., Ishwar, B.,(2006): Linear stability of generalized photogravitational restricted three body problem with PoyntingRobertson drag,Journal of Dynamical system \& Geometric Theories 4(1),79-86.

[5] Liou J. C., Zook, H.A. and Jackson, A.A. (1995b): Radiation pressure,Poynting-Robertson drag and solar wind drag in the restricted three body problem, Icarus 116,186-201. http://dx.doi.org/10.1006/icar.1995.1120.

[6] Murray,C.D.,(1994): Dynamical effects of drag in the circular restricted three body problem:Location and stability of the Lagrangian equilibrium points, Icarus 112,465-484. http://dx.doi.org/10.1006/icar.1994.1198.

[7] Narayan. A. and Kumar,C.R. (2011): Effects of photogravitational and oblateness on the triangular Lagrangian points in the elliptical restricted three body problem,Int.J.Pure and Appl.Math,68,201-224.

[8] Poynting, J.H., (1903): Radiation in the solar system: its effect on temperature and its pressure on small bodies, MNRAS, 64,525-552. http://dx.doi.org/10.1093/mnras/64.1.1a.

[9] Robertson.H.P. (1937): Dynamical effects of radiation in the solar system, MNRAS, 97, 423-438. http://dx.doi.org/10.1093/mnras/97.6.423.

[10] Schuerman, D. (1980): The restricted three body problem including radiation pressure, Astrophys. J., 238, 337-342 http://dx.doi.org/10.1086/157989.

[11] Sahoo, S.K.,Ishwar,B.,(2000): Stability of collinear equilibrium points in the generalized photogravitational elliptic restricted three body problem,Bulletin of the Astronomical Society of India 28,579 586.

[12] Singh, J. and Umar, A.(2012):Motion in the photogravitational elliptic restricted three body problem under an oblate primary, The Astronomical Journal, 143:109,(22pp).

[13] Wyatt S.P and Whipple F.L. (1950): The Poynting-Robertson effect on meteor orbits,American Astron.Soc.APJ.111,134-141. http://dx.doi.org/10.1086/145244.

[14] Zimovshchikov, A.S., Tkhai. (2004): Instability of libration points and resonance phenomena in the photogravitational elliptic restricted three body problem, V.N.: Sol. Syst. Res. 38, 155-164. http://dx.doi.org/10.1023/B:SOLS.0000022826.31475.a7. 